\title{
Preparation and characterisation in simulated body conditions of glutaraldehyde crosslinked chitosan membranes
}

\author{
R. M. SILVA ${ }^{1,2 *}$, G. A. SILVA ${ }^{1,2}$, O. P. COUTINHO ${ }^{2,3}$, J. F. MANO ${ }^{1,2}$, R. L. REIS ${ }^{1,2}$ \\ ${ }^{1}$ Department of Polymer Engineering, University of Minho, Campus de Azurém, 4800-058 \\ Guimarães, Portugal \\ E-mail: ricardosilva@dep.uminho.pt \\ ${ }^{2} 3$ Bs Research Group, Biomaterials, Biodegradables, Biomimetics, Campus de Gualtar \\ 4710-053 Braga, Portugal \\ ${ }^{3}$ Department of Biology, University of Minho, Campus de Gualtar, 4710-053 Braga, Portugal
}

Chitosan membranes, aimed at biomedical applications, were prepared by a solvent casting methodology. Crosslinking was previously performed in acetic acid solution with glutaraldehyde, in order to obtain different degrees of crosslinking. Some membranes were neutralised in a $\mathrm{NaOH}$ solution. Mechanical tensile tests comprised quasi-static experiments at constant stress rate and temperature sweep dynamic mechanical analysis tests. This included measurements with the samples immersed in isotonic saline solution at $37^{\circ} \mathrm{C}$, in order to simulate physiological conditions, that were performed using a specific liquid container. It was observed that for higher crosslinking levels the membranes become stiffer but their strength decreases; these results are in agreement with swelling tests, also performed at body temperature. All the membranes exhibited similar and significant damping properties in wet conditions, which were stable in a broad temperature range. Weight loss measurements showed that the developed membranes degrade slowly up to 60 days. Cytotoxicity screening, using cell culture tests, showed that eventually such materials could be adequate for use in biomedical applications.

(C) 2004 Kluwer Academic Publishers

\section{Introduction}

Chitin is the second most abundant biopolymer in nature and the supporting material of crustaceans, insects, fungi, etc. Chitosan can be obtained by N-deacetylation of chitin, although this $\mathrm{N}$-deacetylation is almost never complete. Thus, chitosan is a co-polymer of 2-amino-2deoxy-D-glucopyranose (GlcN) and 2-acetamido-2deoxy-D-glucopyranose (GlcNAc).

The ratio of GlcN units to both glucopyranose structural units is known as the degree of deacetylation (DD) and has a striking effect on chitosan physical and chemical properties. The DD is typically more than $75 \%$ [1]. The presence of free amino groups that can be protonated at acidic conditions make it soluble in dilute aqueous solutions of formic, acetic, lactic, citric and hydrochloric acid [2]. Chitosan acid-base equilibrium pKa depends on the net charge and its solubility occurs in the range of the degree of protonation of ca. 0.5. Chitosan (DD 88\%) acetate neutral salt possesses a pKa of approx. 6.2 at that degree of protonation [3]. Chitosan can be hydrolysed by lysozyme present in human body fluids [4-7]. The degradation rate of chitosan by lysozyme depends on the DD, reaching a maximum at about $50 \%$.
In turn, no apparent lysozyme degradation was observed in chitosan with DD higher than 97\% [8,9].

This natural polymer is of special interest in the biomedical field, since it displays excellent properties such as biocompatibility, biodegradability, non-toxicity [1] and bioactivity [10], and its degradation products are non-toxic, non-immunogenic and non-carcinogenic [11]. Chitosan has been widely studied and proposed for biomedical applications, namely for skin tissue regeneration, wound dressings, as barrier-membranes to prevent the ingrowth of undesirable connective tissue, sutures and for sustained drug release [1].

Crosslinking chitosan is an appropriate methodology for controlling its swelling rate, drug release rate and changing of mechanical properties. Some crosslinking reagents have been suggested in literature, like glutaraldehyde [12-16], sulphuric acid [17], genipin [18,19] and oxidised glucose [20]. Glutaraldehyde is the most widely studied crosslinker of chitosan and it was often used as a comparison in the study of novel crosslinkers. The reaction with chitosan amine groups produces covalent crosslinking through a Schiff base reaction. Three different propositions are considered: (a) there is

*Author to whom all correspondence should be addressed. 
the formation of one Schiff base and the other aldehyde group of glutaraldehyde remains free (in this case no crosslinking is formed); (b) the crosslinking occurs with one glutaraldehyde molecule and two chitosan unities, involving the formation of two Schiff bases; (c) a great crosslinking chain is formed due to the polymerisation of glutaraldehyde. Chitosan dissolved in acetic acid pH 3-4 react with glutaraldehyde quite quickly, less than $1 \mathrm{~h}$. Therefore, the protonation of chitosan amine groups does not affect the reaction, while the concentration of glutaraldehyde strongly affects the physical properties of the general compounds formed [21]. In this work, chitosan membranes with no, low or high crosslinking degree, as induced by glutaraldehyde were prepared and their degradation and swelling properties evaluated.

The mechanical properties are usually an important criteria in selection of material for biomedical applications. Water content of hydrogel materials can affect drastically its mechanical properties, especially when specimens are in contact with physiological fluids. In this sense, materials should be tested in a water bath or in a high humidity environment. Some authors $[14,18]$ have reported mechanical properties of chitosan membranes crosslinked with glutaraldehyde in dry state. However, some applications require the knowledge of mechanical properties measured in physiological conditions. In this work, an isotonic saline solution (ISS) was used to simulate body fluid conditions and tests were performed with samples immersed at $37^{\circ} \mathrm{C}$.

On the other hand, a material to be used as an implant or as a scaffold for tissue engineering applications must exhibit an appropriate biological behaviour in terms of interaction with living tissues [22]. The first step in the evaluation of these materials in terms of biological behaviour is their cytotoxicity testing in vitro, using immortalised cell lines [23]. Cytotoxicity deals mainly with substances that leach out of the proposed biomaterials [24], which in contact with cells allow for the evaluation of its possible toxicity over cells. Parameters like cell morphology, death, proliferation and adhesion are evaluated for a preliminary screening of the biocompatibility. Biochemical tests such as MTT and total protein quantification are often used in these indirect contact studies. The MTT test has been widely used to measure cellular viability and proliferation [2527], and the total protein test is a fairly accurate measure of cell proliferation [28].

\section{Materials and methods}

\subsection{Membranes preparation}

Chitosan from crab shells was purchased from SigmaAldrich. According to the manufacturer its DD is superior than $85 \%$. Acetic acid, glutaraldehyde and other reagents were of reagent grade.

The chitosan solution was prepared by dissolving chitosan $(1 \mathrm{wt} \%)$ in acetic acid solution (1 wt \%). Glutaraldehyde solutions with different concentrations were prepared diluting $50 \mathrm{wt} \%$ glutaraldehyde solution as it was provided by the manufacturer. The same volume of each glutaraldehyde solution was added to a certain amount of the previous chitosan solution in order to obtain a glutaraldehyde chitosan amine groups molar ratio (assuming the minimum degree of acetylation 85\%) of $1 \%$ (ch01GA), 10\% (ch10GA) and 20\% (ch20GA). Glutaraldehyde solution was added dropwise for $5 \mathrm{~min}$ under gentle stirring and the resultant solutions were allowed to settle for about $1 \mathrm{~h}$ to prevent any air bubble forming. Non-crosslinked chitosan membranes (ch) were prepared in the same way, but no glutaraldehyde solution was added. Membranes were prepared by solvent casting. Solutions were poured into Petri dishes and dried at $37^{\circ} \mathrm{C}$. After drying, membranes were neutralised in $\mathrm{NaOH} 0.1 \mathrm{M}$ solution for $10 \mathrm{~min}$, washed thoroughly with distilled water and dried again at $37^{\circ} \mathrm{C}$. Some samples were non-neutralised to be tested in dry state. Obviously, non-neutralised chitosan membranes cannot be tested in solution, since they rapidly swell and dissolve due to the protonated amine groups.

\subsection{X-ray diffraction}

The morphology of the studied films were analysed by Xray diffraction using synchrotron radiation at the A2 Soft Condensed Matter Beamline of HASYLAB, DESY (Hamburg, Germany). 2-D wide-angle X-ray scattering (WAXS) patterns were obtained employing an image plate, separated $22 \mathrm{~cm}$ from the sample.

\subsection{Degradation and swelling kinetics}

The water uptake measurements were carried out by means of immersing neutralised samples, previously weighted, in $30 \mathrm{ml}$ of ISS $(\mathrm{NaCl} 0.154 \mathrm{M}$ and $\mathrm{pH}=7.4 \pm 0.02)$. Containers were sealed and placed in a thermostatic bath at $37 \pm 1{ }^{\circ} \mathrm{C}$. After $15 \mathrm{~min}$ and 1, 2, 4, 8,24 and $54 \mathrm{~h}$ samples were removed from containers, adsorbed water was removed by sandwiching between two paper towels and weighed immediately. The water uptake $(W)$ of all formulations was calculated using the following equation:

$$
W=\frac{M-M_{0}}{M_{0}}=\frac{M}{M_{0}}-1
$$

where $M_{0}$ is the initial weight of the sample and $M$ is the weight of the sample at certain immersion times.

In vitro degradation of chitosan membranes was assessed again in $30 \mathrm{ml}$ of ISS $(\mathrm{NaCl} 0.154 \mathrm{M}$ and $\mathrm{pH}=7.4 \pm 0.02$ ). Samples were previously weighed and then fully immersed in solution. Containers were sealed and placed in a thermostatic bath at $37 \pm 1{ }^{\circ} \mathrm{C}$. After each test period $(7,14,30$ and 60 days) samples were dried until constant mass $\left(M_{1}\right)$. The weight loss (WL) was calculated using the following equation:

$$
\mathrm{WL}=\frac{M_{0}-M_{1}}{M_{0}}=1-\frac{M_{1}}{M_{0}}
$$

where $M_{0}$ is the initial weight of the sample.

\subsection{Quasi-static mechanical properties}

Chitosan membranes neutralised in $0.1 \mathrm{M} \mathrm{NaOH}$ solution were cut into strips. Their dimensions were found to be typically about $15 \times 2 \times 0.020 \mathrm{~mm}^{3}$ after conditioning in ISS for $15 \mathrm{~min}$. Thickness was taken as a mean of 10 


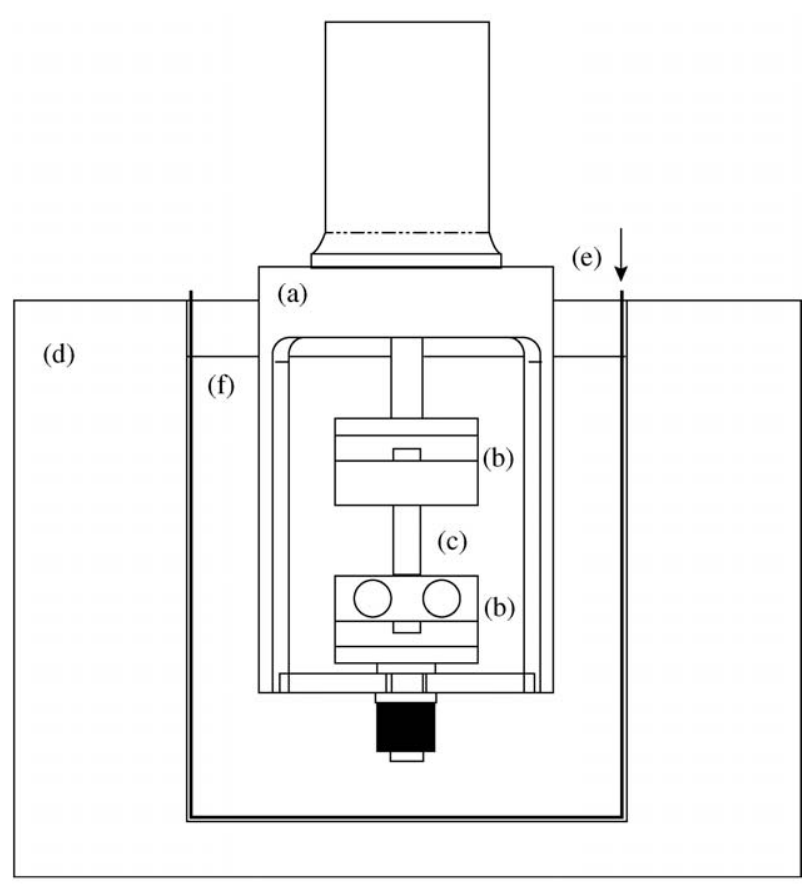

Figure 1 Schematic picture of DMAe7 Perkin-Elmer water bath system, used to test biomaterials in ISS: (a) DMA tensile accessory; (b) top and bottom clamps; (c) sample (membrane); (d) DMA furnace; (e) metallic liquid reservoir; (f) isotonic saline solution.

values at different points measured with a low-pressure micrometer. Their resistance to stretching was evaluated with a Perkin-Elmer DMA7e at a constant stress rate of $5 \mathrm{MPa} / \mathrm{min}$ using the tensile mode. In such experiments, the strain was monitored as a function of stress. Note that such procedure is different from conventional mechanical tests where the stress is monitored as a function of strain, which varies at constant rate. However, one can also build stress-strain curves and obtain a measure of the stiffness (by looking at the slope of the curve at early stages) and the strength (measured by the stress at break) of the sample, when experiments at constant stress rate are performed. The assays with samples immersed in solution were preformed using a liquid bath built in stainless steel. This bath can be fitted into the furnace of the DMA equipment. A schematic representation is shown in Fig. 1.

Mechanical tests in immersed conditions were carried out in an ISS $(\mathrm{NaCl} 0.154 \mathrm{M}$ and $\mathrm{pH}=7.4 \pm 0.02)$ at body temperature $\left(37^{\circ} \mathrm{C}\right)$. Samples were kept immersed for $15 \mathrm{~min}$ in order to reach the hydration and thermal equilibrium, after holding it in the test probe. Mechanical tests in a dry environment and in solution at room temperature (ca. $20^{\circ} \mathrm{C}$ ) were undertaken for comparison purposes. Non-neutralised chitosan membranes were only tested in a dry environment. Temperature was checked with an external temperature sensor after and before each test. The temperature read by the sensor fluctuated less then $0.1^{\circ} \mathrm{C}$ during the experiments. Secant modulus was calculated at $2 \%$ of elongation. Stress and strain at break were also estimated.

\subsection{Dynamic mechanical analysis (DMA)}

Dynamical mechanical measurements were carried out on chitosan membranes with a Perkin-Elmer DMA7e analyser at $1 \mathrm{~Hz}$ and heat rate of $2{ }^{\circ} \mathrm{C} / \mathrm{min}$. The tests were preformed with samples immersed in ISS, using the same system described above. In these measurements, sample and solution was carefully cooled down to near $0^{\circ} \mathrm{C}$, in order to avoid solution freezing and consequent sample damage. Experiments were stopped at about $80^{\circ} \mathrm{C}$. The dimensions were the same as those for quasi-static mechanical tests and thickness was taken by the method described in the previous section. It was considered that the cross-section area of the samples do not vary during the experiment.

During each DMA experiment, both the storage modulus, $E^{\prime}$, and the loss factor, $\tan \delta$, were measured as a function of temperature. The first corresponds to the real component of the complex modulus $\left(E^{*}=E^{\prime}+i E^{\prime \prime}\right)$, being a measure of the sample's stiffness, whereas the later gives the ratio between the amount of mechanical energy lost and stored during a cycle $\left(\tan \delta=E^{\prime \prime} / E^{\prime}\right)$, measuring the damping capability of the sample.

A sample of polybutadiene was tested both into solution and in the conventional dry state. This rubber material was found to not absorb any water by a simple gravimetric assay and so mechanical dynamic performance in wet conditions should not differ from conventional assay. In fact, it was found that both $E^{\prime}$ and $\tan \delta$ are similar when measuring in both dry and wet conditions, indicating that the effect of the solution does not influence significantly the measurements.

\subsection{Cytotoxicity}

The materials were cut in $1 \times 1 \mathrm{~cm}^{2}$ pieces and sterilised by ethylene oxide (EtO), as described before [29]. The sterilisation procedure did not show any adverse effect on the samples (data not shown).

The materials were immersed for $24 \mathrm{~h}$ at $37^{\circ} \mathrm{C}$ (with constant shaking) in DMEM culture media without phenol red (Gibco BRL, USA), supplemented with $10 \%$ foetal bovine serum (FBS) (BioChrome, Germany) and $1 \%$ antibiotic/antimycotic solution (Sigma, St. Louis, USA), at a ratio of $1.5 \mathrm{~cm}^{2} / \mathrm{ml}$. This is an advised procedure for biomaterials extraction to obtain the major leachables and determine their short-term effect in a dynamic environment [23]. The extracts were then filtered through a $0.45-\mu \mathrm{m}$ pore size filter and then used for MTT and total protein quantification tests.

\subsection{MTT test}

The MTT (3-(4,5-dimethylthiazol-2-yl)-2,5-diphenyltetrazolium bromide) has a yellow tonality and is soluble in water. This compound can be converted by the mitochondrial enzyme succinate dehydrogenase in a purple colour salt insoluble in water. The insoluble salt absorbs at a wavelength of $570 \mathrm{~nm}$ and it is proportional to the amount of viable cells, since only viable cells can metabolise MTT [26, 27, 30].

A commercial cell line of rat lung fibroblasts, L929, was used in these studies. The cells were cultured in $75 \mathrm{~cm}^{2}$ flasks (Costar). After detaching them from the culture flask by using a $0.25 \%$ trypsin-EDTA solution (SIGMA, St. Louis) they were re-suspended in DMEM culture medium. The cells were plated in 96-well micrometer plates at a density of $2 \times 10^{4}$ cells/well. 
The plates were incubated for $48 \mathrm{~h}$ in a humidified atmosphere of $5 \% \mathrm{CO}_{2}$ at $37^{\circ} \mathrm{C}$. After this, the culture medium was removed and the extracts were placed in contact with the cell monolayer and culture medium was used as control. The plates were incubated for $72 \mathrm{~h}$, and after removal of the culture medium, $50 \mu \mathrm{l}$ of MTT (Sigma, St. Louis, USA) solution $(1 \mathrm{mg} / \mathrm{ml}$ in culture medium) was added and incubated $4 \mathrm{~h}$ at $37^{\circ} \mathrm{C}$. To dissolve the formazan crystals that are formed, $100 \mu \mathrm{l}$ of isopropanol were added and the plates were incubated for 15 minutes at $37^{\circ} \mathrm{C}$ and then placed at room temperature in an orbital shaker to help dissolve the crystals. The optical densities at 570 and $650 \mathrm{~nm}$ (background) were read on a multiwell plate reader (Molecular Dynamics, Amersham, USA) against a blank of MTT solution and isopropanol. All the materials were tested in 10 replicates for each extract for at least three separate experiments with reproducible results.

\subsection{Total protein quantification}

The method used to quantify the total protein make use of the Micro BCA Protein Assay Reagent Kit (Pierce, USA) that uses bicinchoninic acid (BCA) as the detection reagent for $\mathrm{Cu}^{1+}$, which is formed when $\mathrm{Cu}^{2+}$ is reduced by proteins in an alkaline environment. The purple colour product is formed by the chelation of two molecules of BCA with one $\mathrm{Cu}^{1+}$ ion. This complex is water-soluble and absorbs at $562 \mathrm{~nm}$, and its absorbance is linearly correlated with protein concentration [28].

The procedure is very similar to the one of MTT. After $72 \mathrm{~h}$ incubation, the extracts are removed from contact with cells and these are washed with a phosphate buffer solution (PBS) $0.01 \mathrm{M}$, and $100 \mu 1$ of PBS $0.01 \mathrm{M}$ are added to each well. To each well $100 \mu \mathrm{l}$ of the BCA reagent were added, the plates were agitated for $30 \mathrm{~s}$ and incubated for $2 \mathrm{~h}$ at $37^{\circ} \mathrm{C}$. The plates were then cooled to room temperature and the optical densities were measured at $562 \mathrm{~nm}$ against a blank of PBS $0.01 \mathrm{M}$ and BCA reagent. Total protein (in $\mu \mathrm{g} / \mathrm{ml}$ ) was determined using a BSA standard curve.

\section{Results and discussion}

\subsection{Crystallinity}

The 2-D WAXS pattern of non-crosslinked chitosan (not shown) exhibited a Debye ring at $2 \theta \approx 20^{\circ}$ and an isotropic crystalline orientation. This reflection is in agreement with the typical chitosan diffractogram [21,31]. The equatorial cuts of such patterns for the studied chitosan membranes are shown in Fig. 2. One clearly sees that for $1 \%$ crosslinking the crystallinity degree is highly depressed. The results for highercrosslinked materials show that these membranes are amorphous.

\subsection{Degradation and swelling kinetics}

All samples reached the equilibrium hydration degree in about $15 \mathrm{~min}$. This can be observed in Fig. 3, which shows the swelling isotherms and respective $\mathrm{pH}$ drift. Chitosan membranes with lower crosslinking degree (ch01GA) presented higher equilibrium hydration degree

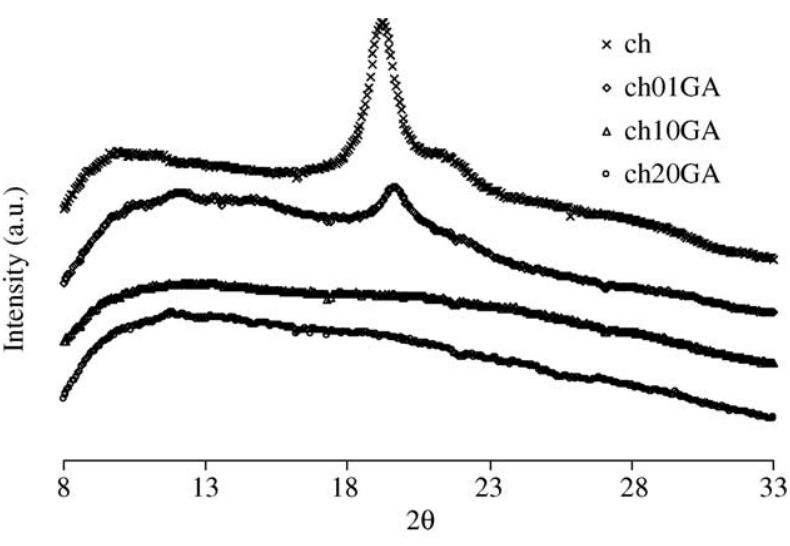

Figure 2 X-ray diffraction patterns of neutralised chitosan membranes non-crosslinked (ch) and with crosslinker chitosan amine groups molar ratio of $1 \%$ (ch01GA), 10\% (ch10GA) and 20\% (ch20GA).

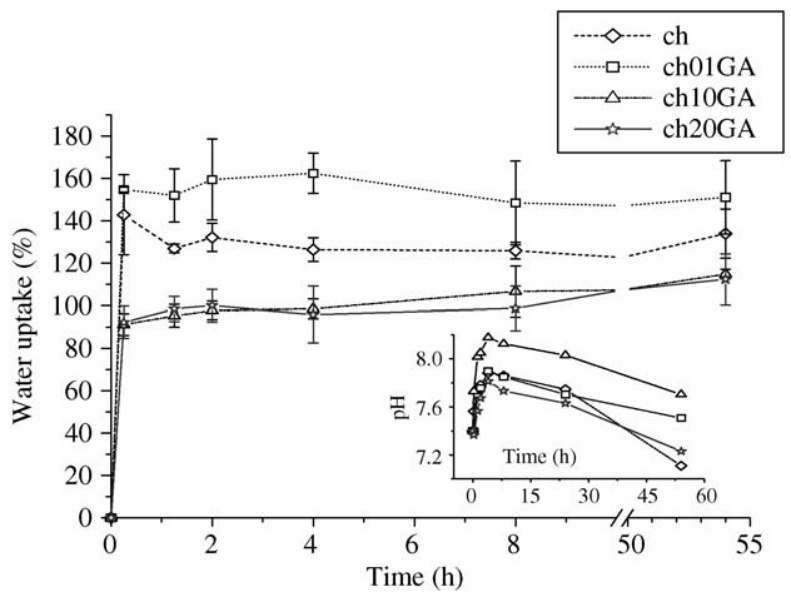

Figure 3 Swelling isotherms at $37^{\circ} \mathrm{C}$ in ISS $(\mathrm{NaCl} 0.154 \mathrm{M}$ and $\mathrm{pH}=7.4 \pm 0.02$ ) of chitosan membranes (ch) and with crosslinker chitosan amine groups molar ratio of $1 \%$ (ch01GA), 10\% (ch10GA) and $20 \%$ (ch20GA). Variation of $\mathrm{pH}$ during tests (inset graphics). Data represents mean \pm standard deviation of at least three samples.

than non-crosslinked membranes (ch) despite the crosslinking reactions. On the other hand, higher crosslinking degrees (ch10GA and ch20GA) showed to be effective on lowering the equilibrium hydration degree. In fact, crystallinity decreases as crosslinking degree by glutaraldehyde increases, since crosslinks between two chitosan units or pendant glutaraldehyde with one aldehyde free may constitute an obstacle to chitosan molecules packing. The lower crystallinity enhances the water molecules accessibility. Thus, the hydration equilibrium degree should depend on the balance between crystallinity and crosslinking degree leading to a maximum somewhat between non-crosslinked membranes (ch) and ch10GA.

All formulations revealed to be very stable in the simulated body wet environment with a weight loss never superior than $12 \%$ after 60 days of immersion. Membranes degrade up to 30 days of immersion. After that, samples weight seems to stabilise and weight loss reach what can be roughly described as a plateau. These results may suggest that the low molecular mass fraction of the chitosan membranes is continuously released in the first 30 days. The higher molecular mass components 


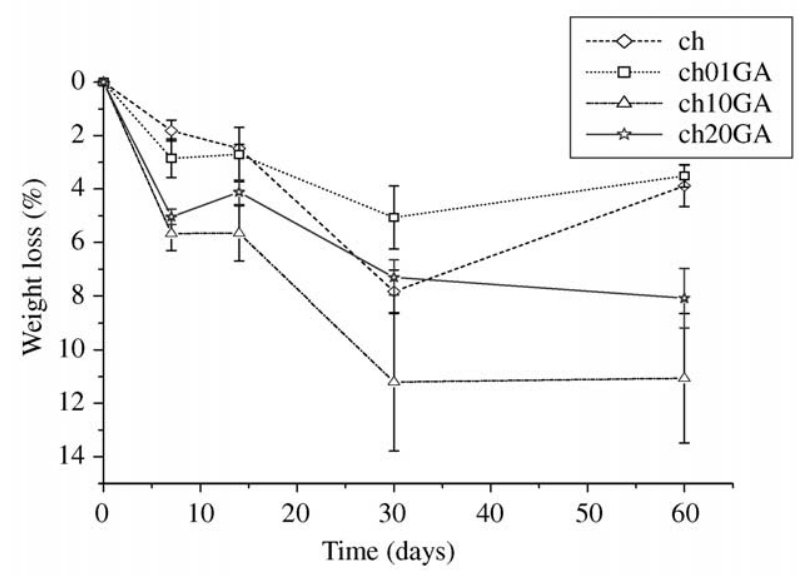

Figure 4 Degradation isotherms at $37^{\circ} \mathrm{C}$ in ISS $(\mathrm{NaCl} 0.154 \mathrm{M}$ and $\mathrm{pH}=7.4 \pm 0.02$ ) of chitosan membranes (ch) and with crosslinker chitosan amine groups molar ratio of $1 \%$ (ch01GA), $10 \%$ (ch10GA) and $20 \%$ (ch20GA). Data represents mean \pm standard deviation of at least three samples.

are much more stable within the experimental time window studied.

The results in Fig. 4 shows that the weight loss can be despised when performing mechanical tests on such membranes in wet state, since the time scale of such experiments is relatively short (only a few minutes) when compared to the time required to reach a significant weight loss.

\subsection{Quasi-static mechanical properties}

The results obtained for assays performed in dry state are presented in Table I. No significant differences were found between neutralised and non-neutralised chitosan membranes for evaluated tensile mechanical properties in the dry state. Similar values of non-neutralised chitosan films have been reported by Bégin et al. [2]. In order to evaluate the mechanical performance of the several prepared formulations in wet state, tensile tests were carried out in ISS. It was impossible to test membranes with a higher degree of crosslinking (ch20GA), because of its low resistance in wet state.

The mechanical resistance to stretching decreased sharply, when samples were swollen in the ISS. Secant modulus decreased about 100 -fold from $1.8 \mathrm{GPa}$ to $13 \mathrm{MPa}$ at room temperature, while stress at break decreased about 10-fold from 59 MPa to 6.1 MPa at the same temperature. This is a consequence of the high equilibrium swelling degree. On the other hand, strain at failure increased from 4.9 to $59 \%$ due to the water plasticising effect. Typical stress-strain curves of samples tested as well as the corresponding relevant mechanical properties for all conditions studied are shown in Figs. 5 and 6, respectively.

Secant tensile modulus decreased when a small amount of glutaraldehyde was used to crosslink chitosan (ch01GA), for both temperatures tested. On the other hand, modulus increase for higher concentrations (ch10GA). The minimum in stiffness found at ch01GA can be related with the maximum achieved for the equilibrium hydration degree for the same sample (Fig. 3). However, a similar result was obtained for ch01GA tested in a dry environment (results not showed). In this
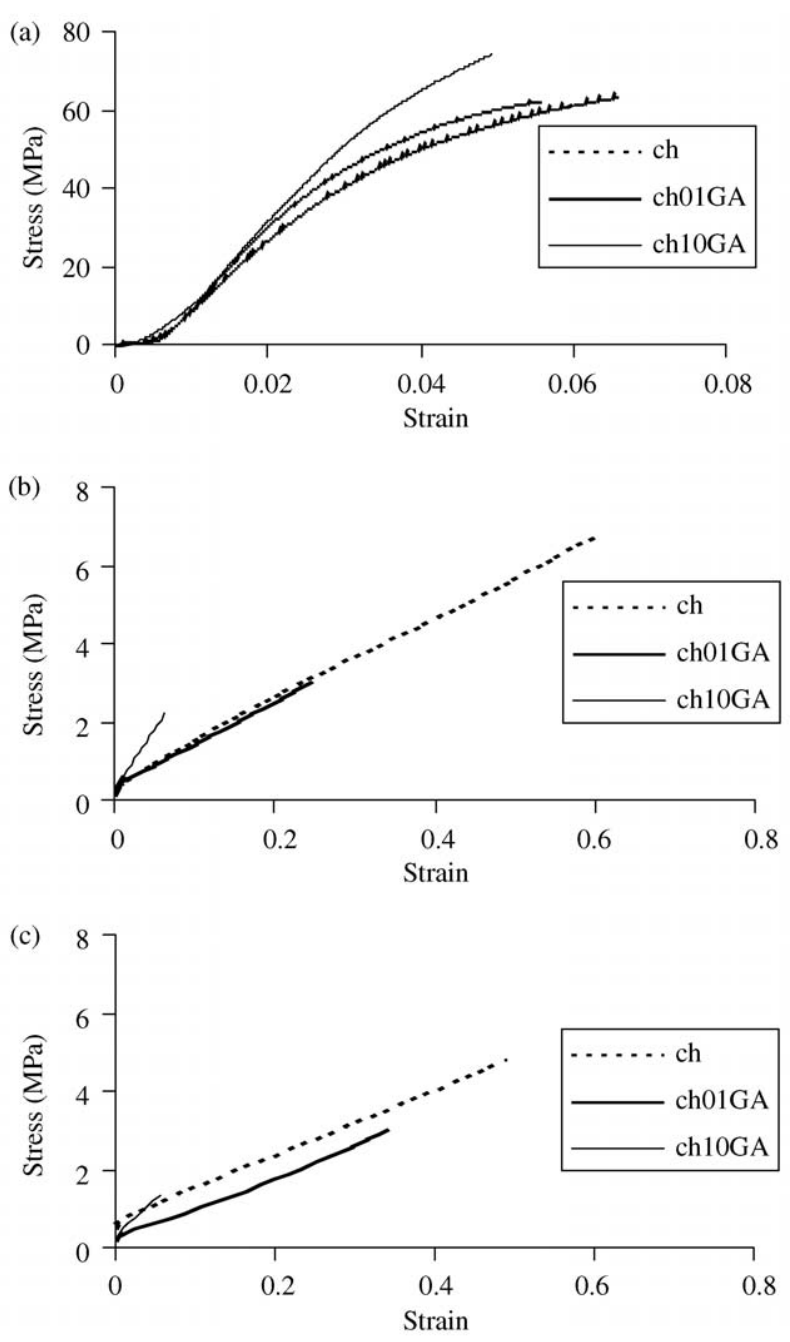

Figure 5 Typical stress-strain curves of membranes of non-crosslinked chitosan (ch) and with crosslinker chitosan amine groups molar ratio of $1 \%$ (ch01GA) and 10\% (ch10GA): (a) neutralised membranes tested in dry state at room temperature; (b) neutralised membranes tested in ISS at room temperature; (c) neutralised membranes tested in ISS at $37^{\circ} \mathrm{C}$.

way, the balance between the effects of opposite variation of crystallinity and crosslinking degree may also have an effect on this behaviour, besides the hydration degree. It is well known that the elastic modulus increase when reducing the average molecular weight between crosslinks. However, in such a complex system where a semi-crystalline polymer is crosslinked with glutaraldehyde, which possesses a distinct chemical character from the original polymer, crystallinity, hydration degree and different hydrophilicity can also play a certain role. In this way, it is very difficult to correlate all those factors with the final viscoelastic properties of such materials.

At physiologic temperature the modulus decreased for each sample tested, relative to what was observed at room temperature. Moreover, stress and strain at failure decreased when the crosslinking degree increased, since samples become fairly brittle for glutaraldehyde chitosan amine groups molar ratio of $10 \%$ (ch10GA).

\subsection{Dynamic mechanical analysis}

The storage modulus of non-crosslinked chitosan membranes (ch) was almost constant during temperature 
T A B L E I Mechanical tensile properties of non-crosslinked chitosan (ch) membranes (average \pm standard deviation of at least three samples) performed in the dry state

\begin{tabular}{lcll}
\hline & $E_{2 \%}(\mathrm{GPa})$ & $\sigma_{\text {at break }}(\mathrm{MPa})$ & $\varepsilon_{\text {at break }}(\%)$ \\
\hline Non-neutralised & $1.46 \pm 0.63$ & $51.7 \pm 12.5$ & $5.85 \pm 0.45$ \\
Neutralised & $1.84 \pm 0.33$ & $58.7 \pm 11.0$ & $4.85 \pm 1.25$ \\
\hline
\end{tabular}

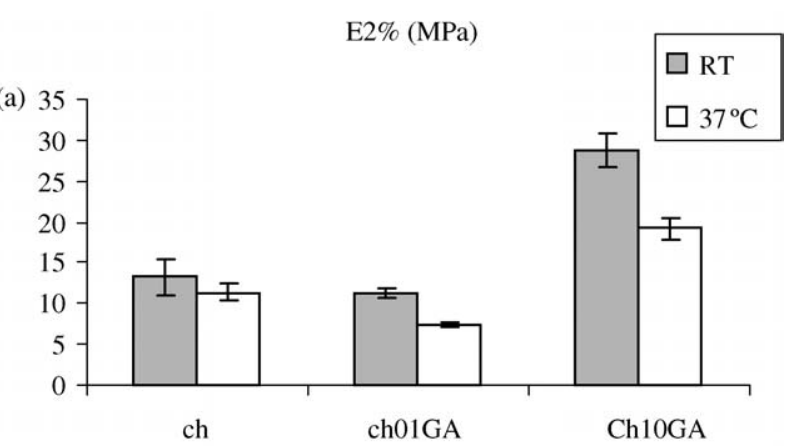

Stress at break (MPa)

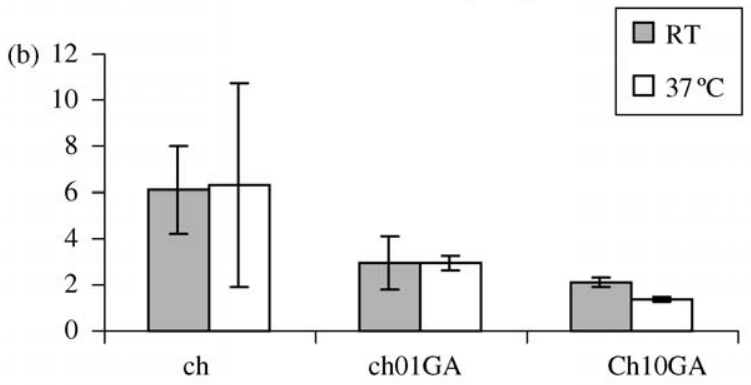

Strain at break $(\%)$

(c) 100

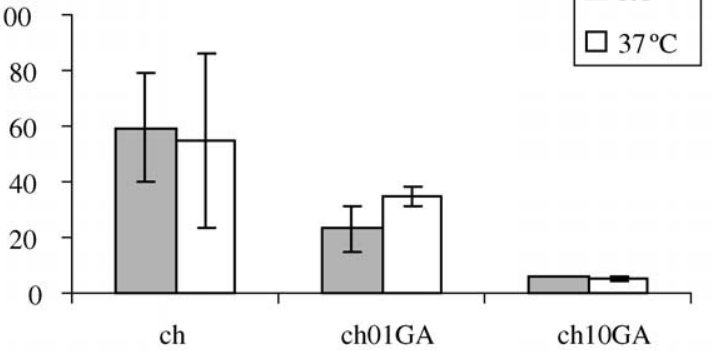

Figure 6 Tensile properties of neutralised chitosan membranes noncrosslinked (ch) and with crosslinker chitosan amine groups molar ratio of 1\% (ch01GA) and 10\% (ch10GA), measured in ISS: (a) secant modulus at $2 \%$ of elongation; (b) stress at break; (c) strain at break. Data represents mean \pm standard deviation of at least three samples.

scan, only showing a slight decrease around $40^{\circ} \mathrm{C}$ (Fig. 7.a). On the other hand, both crosslinked samples (ch01GA and ch10GA) storage modulus presented a continuous decrease with increasing temperature (Fig. 7.a and 7.b). This is in agreement with a previously described decrease of the secant tensile modulus observed from room to physiological temperature. The slope of such curves seems to increase with crosslinking. All modulus values are quite similar to the values achieved for quasi-static tests and the same trends are basically observed.

The damping properties of the studied membranes are also depicted in Fig. 7, as measured by the loss factor, $\tan \delta$. One founds values between 0.15 and 0.20 , indicating a clear viscoelastic behaviour for the prepared
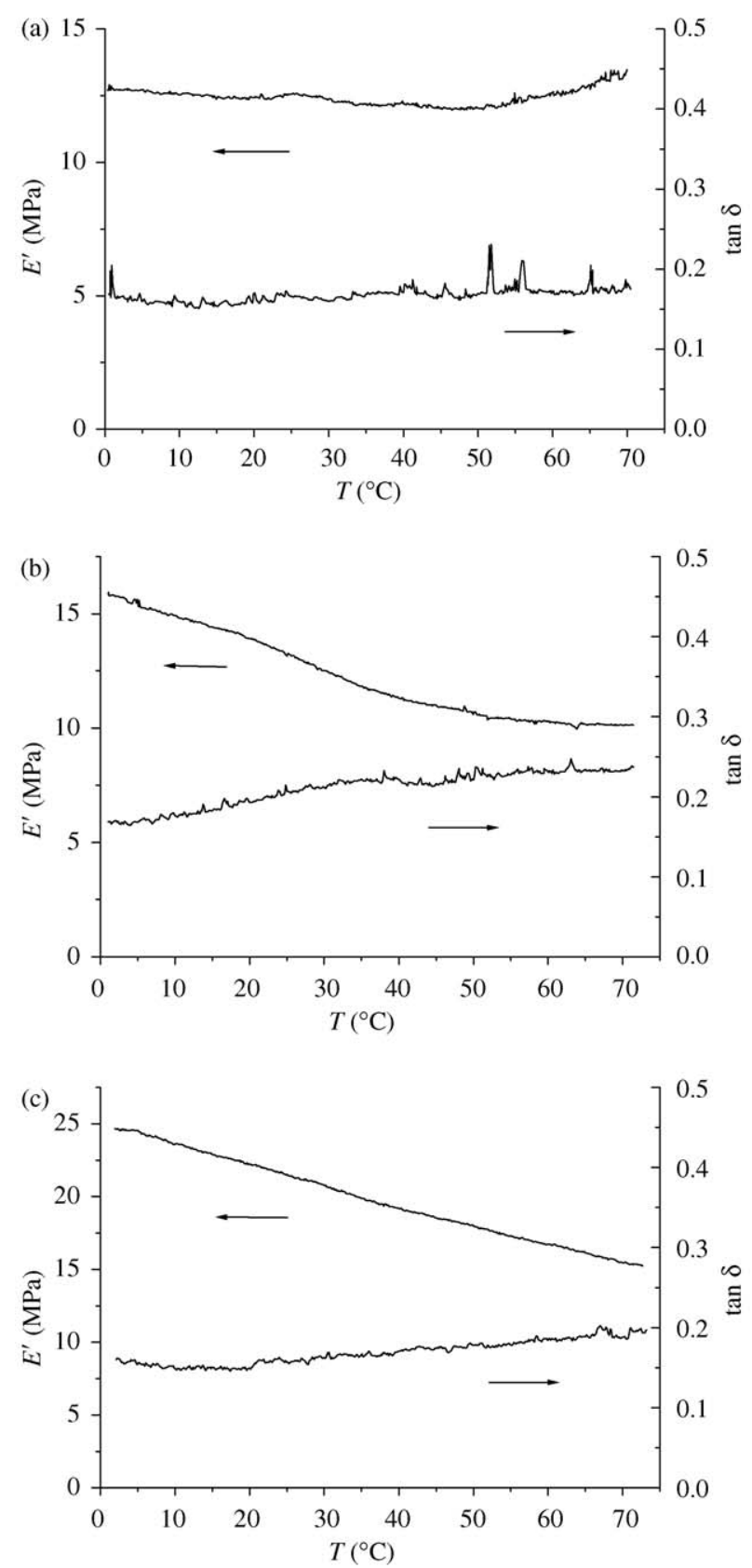

Figure 7 Storage modulus, $E^{\prime}$, and loss factor, $\tan \delta$, at $1 \mathrm{~Hz}$ of neutralised chitosan membranes non-crosslinked (ch) (a), with crosslinker chitosan amine groups molar ratio of $1 \%$ (ch01GA) (b) and 10\% (ch10GA) (c), measured in ISS.

chitosan membranes. This result is in accordance to the viscoelastic character also observed in meshes of chitosan fibres also analysed in wet conditions both by DMA and creep experiments [32]. It should be noticed in this context that most of the living tissues have viscoelastic properties. Thus, besides the conventional quasi-static mechanical properties, new materials to be used in biomedical applications should have compatible time-dependent mechanical (viscoelastic) features with the organs or tissues that they will contact with. Tests such as DMA experiments in simulated physiological conditions, as presented in this work, may constitute a valuable tool for this evaluation.

The results in Fig. 7 indicates that crosslinking do not influence significantly the damping properties of the 
T A B L E I I MTT test and total protein quantification of neutralised chitosan membranes non-crosslinked (ch) and with crosslinker chitosan amine groups molar ratio of $1 \%$ (ch01GA), 10\% (ch10GA) and $20 \%$ (ch20GA)

\begin{tabular}{llc}
\hline Sample & $\begin{array}{l}\text { MTT value }(\%)^{*} \\
\pm \text { Standard deviation }\end{array}$ & $\begin{array}{c}\text { Total protein }(\mu \mathrm{g}) \\
\pm \text { Standard deviation }\end{array}$ \\
\hline Control $^{\dagger}$ & - & $105.5 \pm 22.9$ \\
ch & $74.9 \pm 9.7$ & $126.6 \pm 23.9$ \\
ch01GA & $74.0 \pm 9.3$ & $87.8 \pm 13.5$ \\
ch10GA & $75.4 \pm 7.5$ & $129.7 \pm 15.8$ \\
ch20GA & $67.7 \pm 9.9$ & $132.0 \pm 17.0$ \\
\hline
\end{tabular}

*Viability related to the control, which scored $100 \%$.

${ }^{\dagger}$ Control: cells with culture medium.

chitosan membranes. Moreover, for all formulations analysed, $\tan \delta$ is almost temperature independent, indicating that no physical transition nor relaxation process take place within this temperature range

\subsection{Cytotoxicity}

Cells cultured in contact with the extracts of the materials after $72 \mathrm{~h}$ displayed normal morphology when observed in an inverted light microscope. The results for the MTT test performed after $72 \mathrm{~h}$ of contact of the extraction medium with cells are shown on Table II, and allowed to assay, though qualitatively, the cellular viability. The percentage of cell viability plotted is related to the control, considering the control (cells with DMEM with $1 \%$ antibiotic/antimycotic and $10 \% \mathrm{FBS}$ ) to have $100 \%$ viability.

All the samples present a viability percentage around $80 \%$, which means that these materials do not exert a cytotoxic effect over the cells. It seems that growing concentrations of glutaraldehyde tend to have a negative effect over cell viability, although for the tested concentrations cell viability is maintained at a very good percentage (see Table II). It is known from previous cytotoxic studies performed that increasing the glutaraldehyde concentration increases the cytotoxicity of the membranes (data not shown). Moreover, a similar trend was reported for collagen crosslinked with glutaraldehyde [33]. The results obtained can be considered as a good indicator of the biocompatibility of the tested materials, since it is known that for biodegradable polymers their degradation tends to induce a severe cytotoxic effect due to a $\mathrm{pH}$ drop (as for instance for polylactides and polyglycolides) [23].

As for the total protein quantification, Table II shows that only the cells in contact with the extract of the sample containing $1 \%$ glutaraldehyde as crosslinking agent (ch01GA) displayed a slight smaller total protein content than the control. The other three samples (ch, ch10GA and ch20GA) displayed an even higher amount of total protein than the control. A possible explanation for this result is that the crosslinking strength of the membranes crosslinked with $1 \%$ of glutaraldehyde (ch01GA) is lower than in the other conditions were glutaraldehyde is used as a crosslinker and, in this way, more leachables are obtained during the extraction in DMEM culture medium. So, the membranes noncrosslinked (ch) would not leach components resulting from reaction with glutaraldehyde, while membranes crosslinked with $10 \%$ and $20 \%$ of glutaraldehyde (ch10GA and ch20GA) would not leach those products in such an extent as in the formulation ch01GA. In being so, the leachables of the formulation ch01GA will somehow inhibit the cellular activity, quantified by the protein production, but not diminishing their viability, as seen by MTT test.

The relation between the two tests (MTT and total protein quantification) is not, of course, straightforward, but some conclusions can be drawn from the comparison. The MTT test shows that all the conditions display similar viability patterns, around $80 \%$ when compared with the control, with the exception of the formulation ch20GA (a result in accordance with previous results obtained), that displays a level of $70 \%$ of viability. However, the total protein quantification shows that some of the conditions presented higher protein content than the control itself. This result can be explained by the fact that some of the leachables might in fact stimulate the activity of cells. In this way, the results between the two tests are perfectly complementary and coherent. Although further biocompatibility tests are needed, it is possible to say that the tested membranes have shown to be suitable for biomedical applications.

\section{Conclusions}

Different chitosan-based membranes could be developed. A method was implemented to test tensile mechanical properties of chitosan-based membranes in ISS and $37^{\circ} \mathrm{C}$, in order to simulate body conditions. The mechanical resistance to stretching decreased sharply when swollen in an aqueous environment, mainly due to the high hydration equilibrium degree of chitosan materials. However, neutralised chitosan membranes and membranes crosslinked with small amounts of glutaraldehyde become more flexible, making them suitable for some biomedical applications, that could be wound dressing or as a barrier-membranes keeping space for bone regeneration and preventing the ingrowth of undesirable connective tissue. Moreover, DMA in temperature scan mode confirmed the mechanical stiffness dependence on the temperature of crosslinked chitosan membranes. This dependence increased with the crosslinking degree. All formulations were found to be relatively stable in ISS up to 60 days. In fact, the weight loss was never superior than $12 \%$ and the membranes have kept its mechanical integrity.

All membrane formulations exhibit a viscoelastic behaviour which could have advantages in the mechanical compatibility with the tissues to be repaired.

The cell culture cytotoxicity studies performed indicate that there is a good interaction of the tested materials with the cells. The biochemical tests confirm that the viability of cells in contact with the extracts is maintained. In some cases an increased biochemical activity of cells is observed, which can be correlated indirectly with cell proliferation. In fact, the biological performance of the membranes seems to indicate that they can be used on the proposed biomedical applications, although further cytocompatibility studies must be 
carried out before moving into any type of animal experimentation.

\section{Acknowledgments}

R. M. Silva was supported by the Ph.D. Scholarship SFRH/BD/6862/2001 from the Portuguese Foundation for Science and Technology (FCT) under the POCTI programme. This work was partially supported by FCT through funds from the POCTI and/or FEDER programmes.

\section{References}

1. M. N. V. RAVI KU MAR, React. Funct. Polym. 46 (2000) 1.

2. A. BÉGIN and M.-R. V. CALSTEREN, Int.J. Biol.Macromol. 26 (1999) 63.

3. M. Rinaudo, G. PAVlov and J. Desbrières, Polymer 40 (1999) 7029.

4. K. M. VARUM, M. M. MYHR, R. J. N. HJERDE and O. SMIDSROD, Carbohyd. Res. 299 (1997) 99.

5. K. M. VARUM, H. K. HOLME, M. IZUME, B. T. STOKKE and O. SMIDSROD, Biochim. Biophys. Acta Gen. Subj. 1291 (1996) 5 .

6. S. AIB A, Carbohyd. Res. 261 (1994) 297.

7. R. J. NORDTVEIT, K. M. VARUM and O. SMIDSROD, Carbohyd. Polym. 29 (1996) 163.

8. K. KURITA, Y. KAJI, T. MORI and Y. NISHIYAMA, ibid. 42 (2000) 19.

9. K. TOMIHATA and Y. IKA DA, Biomaterials 18 (1997) 567.

10. K. K URITA, Prog. Polym. Sci. 26 (2001) 1921.

11. R. A. A. Muzzarelli, Carbohyd. Polym. 20 (1993) 7.

12. S. SRIDHAR, G. SUSHEELA, G. J. REDDY and A. A. KHAN, Polym. Int. 50 (2001) 1156.

13. B. KRAJEWS KA, J. Chem. Technol. Biotechnol. 76 (2001) 636.

14. M. V. RISBUD and S. V. BHAT, J. Mater. Sci.: Mater. Med. 12 (2001) 75 .

15. D. A. MUSAlE and A. KUMAR, Sep. Purif. Technol. 21 (2000) 27.
16. X. P. WANG, Y. F. FENG and Z. Q. SHEN, J. Appl. Polym. Sci. 75 (2000) 740.

17. J. GE, Y. CUI, Y. YAN and W. JIANG, J. Membr. Sci. 165 (2000) 75 .

18. F.-L. MI, Y.-C. TAN, H.-C. LIANG, R.-N. HUANG and H.-W SUNG, J. Biomater. Sci. Polymer Edn 12 (2001) 835.

19. F.-L. Mi, H.-W. SUng and S.-S. ShyU, J. Polym. Sci. Pol. Chem. 38 (2000) 2804.

20. F. LI, W. G. LIU and K. D. YAO, Biomaterials 23 (2002) 343.

21. O. A. C. MONTEIRO JR. and C. Airoldi, Int. J. Biol. Macromol. 26 (1999) 119.

22. S. C. MENDES, R. L. REIS, Y. P. BOVELL, A. M. CUNHA, C. A. VAN BLITTERSWIJK and J. D. DE BRUIJN, Biomaterials 22 (2001) 2057.

23. A. P. MARQUES, R. L. REIS and J. A. HUNT, ibid. 23 (2002) 1471.

24. M. E. GOMES, R. L. REIS, A. M. CUNHA, C. A. VAN BLITTERWIJK and J. D. DE BRUIJN, ibid. 22 (2001) 1911.

25. A. J. SAlgADo, M. E. GOMES, A. ChOU, O. P. COUTINHO, R. L. REIS and D. W. HUTCHMACHER, Mater. Sci. Eng. C 20 (2002) 27.

26. T. MOS MAN N, J. Immunol. Meth. 65 (1983) 55.

27. T. F. Slater, B. SAWYER and U. STRAUli, Biochim. Biophys. Acta. 77 (1963) 383.

28. P. K. SMITH, Anal. Biochem. 150 (1985) 76.

29. R. L. REIS, S. C. MENDES, A. M. CUNHA and M. BEVIS, Polym. Int. 43 (1997) 347.

30. N. D. WINBLADE, H. SCHMOKEL, M. BAUMANN, A. S HOFFMAN and J. A. HUBBELL, J. Biomed. Mater. Res. 59 (2002) 618.

31. K. T. HWANG, J. T. KIM, S. T. JUNG, G. S. CHO and H. J. PAR K, J. Appl. Polym. Sci. 89 (2003) 3476.

32. K. TUZLAKOĞLU, C. M. ALVES, J. F. MANO and R.L. REIS, Macromol. Biosci., accepted.

33. C. A. SCOTCHFORD, M. G. CASCONE, S. DOWNES and P. GIUSTI, Biomaterials 19 (1998) 1.

Received 10 April 2003

and accepted 18 March 2004 